[0212-7199 (2007) 24: 7; pp 324-327] ANALES DE MEDICINA INTERNA Copyright (C) 2007 ARAN EDICIONES, S.L.

An. Med. InTERnA (Madrid) Vol. 24, N. ${ }^{\circ}$, pp. $324-327,2007$

\section{Lipoproteína (a) en una población urbana de Venezuela: evidencia de que su incremento por deprivación estrogénica es transitorio}

\author{
V. BERMÚDEZ PIRELA, M. CABRERA DE BRAVO, E. MENGUAL MORENO, \\ C. CANO PONCE, E. LEAL GONZÁLEZ, M. LEMUS ANTEPAZ, A. AMELL DE \\ DÍAZ, L. SORELL GÓMEZ \\ Centro de Investigaciones Endocrino-Metabólicas “Dr. Félix Gómez”. Facultad de \\ Medicina. Universidad del Zulia. Maracaibo, Venezuela. Centro de Ingeniería Genética y \\ Biotecnología de La Habana, Cuba
}

\section{RESUMEN}

Objetivo: La lipoproteína (a) se considera un factor de riesgo para enfermedad cardiovascular. En Venezuela se desconoce su comportamiento por lo que el objetivo de este estudio fue determinar la concentración de lipoproteína (a) en individuos de la ciudad de Maracaibo.

Métodos: 500 individuos sanos que acudieron al Centro de Investigaciones Endocrino-Metabólicas, a los que se les cuantificó la lipoproteína (a) por ELISA.

Resultados: La lipoproteína (a) no mostró diferencias significativas ni por edad ni sexo en la población general. En el grupo de mujeres de 40-44 años se observó una tendencia a niveles mayores, por lo que el género femenino se dividió en dos grupos: a) < 40 años (mediana: 13 $\mathrm{mg} / \mathrm{dl}) ; \mathrm{y}$ b) 40 años y más (mediana: $16 \mathrm{mg} / \mathrm{dl}) ;(\mathrm{p}<0,02)$. Al descomponer el grupo de 40 y mas años según recibir terapia de reemplazo hormonal o no, aquellas que recibieron terapia de reemplazo hormonal siempre exhibieron una concentración de lipoproteína (a) menor; $(\mathrm{p}<0,01)$, salvo en el grupo de los 60-64 años, donde no hubo diferencia.

Conclusiones: La lipoproteína (a) en la muestra estudiada se encuentra dentro de los límites considerados como normales. La terapia de reemplazo hormonal disminuye la lipoproteína (a) en mujeres menopáusicas, sin embargo, en las mujeres sin terapia de reemplazo hormonal, la lipoproteína (a) experimenta una disminución progresiva para alcanzar niveles idénticos a aquellas con terapia de reemplazo hormonal en el grupo de 60-64 años.

PALABRAS CLAVE: Lipoproteína (a). Factor de riesgo. Terapia de reemplazo hormonal. Apo (a). Activador tisular del plasminógeno.
LIPOPROTEIN (a) IN AN URBAN POPULATION OF VENEZUELA: EVIDENCE THAT ESTROGENIC DEPRIVATION INCREASE IN LIPOPROTEIN (A) LEVELS IS TRANSITORY

ABSTRACT

Background: Lipoprotein (a) [Lp (a)] is an independent risk factor for coronary artery disease and normal serum levels of this particle is not known in our country. Thus, the aim of this study was to determine plasma Lp (a) concentration in a population sample of Maracaibo.

Patients and method: Fifth hundred out-patients, consulting at Centro de Investigaciones Endocrino-Metabólicas “Dr. Félix Gómez” were randomly underwent to venipunction to obtain a fasting blood simple to assess Lp (a) by a ELISA assay.

Results: No significantly differences were found when compared by sex or age separately, higher levels in Lp (a) was found in female 40-44 year group (median: 20,9 mg/dl). Thus, female population was divided in two sub-groups: < 40 years (median: $13 \mathrm{mg} / \mathrm{dl}$ ) $40 \mathrm{yr}$ and more (median: $16 \mathrm{mg} / \mathrm{dl})$, finding higher Lp (a) levels in the second group $(p<0,02)$. Hormonal replace therapy was assessed by age, resulting that women subjected this approach shows lower levels of $L p(a)(p<0,01)$, except in 60-64 year group.

Conclusions: Lp (a) in a Maracaibo was within normal levels. Hor monal replace therapy diminishes $L p(a)$ concentration in menopausal women, but in menopausal women without hormonal therapy $\mathrm{Lp}(\mathrm{a})$ levels experienced a sustained decrease to normal levels in a age-depended manner.

KEY WORDS: Lipoprotein (a). Risk factor. Hormonal replacement therapy. Apo (a). Tissue plasminogen activator.

Bermúdez Pirela V, Cabrera de Bravo M, Mengual Moreno E, Cano Ponce C, Leal González E, Lemus Antepaz M, Amell de Díaz A, Sorell Gómez L. Lipoproteína (a) en una población urbana de Venezuela: evidencia de que su incremento por deprivación estrogénica es transitorio. An Med Interna (Madrid) 2007; 24: 324-327.

\section{INTRODUCCIÓN}

Inicialmente aislada por Berg del plasma humano en 1963, la lipoproteína (a) [Lp (a)] comparte características estructurales con la lipoproteína de baja densidad (LDL) en cuanto su composición lipídica y presencia de apoproteína B-100 (apo
B-100), pero la presencia de la apoproteína (a) ligada a la apo B-100 mediante una unión covalente hace a la Lp (a) una partícula diferente a la LDL (1). La Lp (a) es una lipoproteína de especial interés por su vinculación al fenómeno aterogénico, ya que se considera un factor de riesgo independiente para el desarrollo enfermedad vascular aterotrombótica (1) y a diferencia

Trabajo aceptado: 14 de marzo de 2007 
del resto de las lipoproteínas aterogénicas, su nivel en sangre está regulado genéticamente, por lo que factores como la dieta y el ambiente tienen poco impacto en su concentración (2).

En Venezuela no existen estudios previos que describan el comportamiento de esta lipoproteína en la población general, por lo que el objetivo del presente trabajo fue determinar los niveles de Lp (a) en una muestra de individuos de la Ciudad de Maracaibo, Estado Zulia, Venezuela.

\section{MATERIALES Y MÉTODOS}

Para la realización de este estudio se seleccionaron al azar 500 individuos que acudieron al Centro de Investigaciones Endocrino-Metabólicas "Dr. Félix Gómez" de la Facultad de Medicina de la Universidad del Zulia, a los que previo consentimiento informado se les realizó una historia clínica completa a fin de descartar procesos patológicos agudos o crónicos que pudiesen influir en los niveles de Lp (a) ya que ésta se comporta como un reactante de fase aguda (2).

Cuantificación de la Lp (a). Previo ayuno de 12 horas cada individuo fue sometido a toma de muestra sanguínea por venipunción la cual fue procesada para obtener suero en el que se determinó la concentración de Lp (a) por el método ELISA de doble anticuerpo (3) (Heber Biotech, La Habana, Cuba).

Análisis estadístico. El comportamiento de la distribución de la Lp (a) se evaluó mediante la prueba $\mathrm{Z}$ de KolmogorovSmirnov, resultando en una distribución no normal, por lo que los resultados se muestran como medianas. Las comparaciones entre grupos se realizaron con la prueba U de Mann-Whitney o ANOVA de un factor (previa conversión logarítmica y prueba post hoc de Tukey) según el caso, considerándose significativo un valor de $\mathrm{p}<0,05$.

\section{RESULTADOS}

Los 500 individuos de la Ciudad de Maracaibo estudiados presentaron niveles de Lp (a) con una mediana de $11,5 \mathrm{mg} / \mathrm{dl}$. No se evidenciaron diferencias significativas al evaluar la concentración sérica de la Lp (a) por género al considerar todas las edades, siendo el valor de la mediana para el sexo femenino de $11,8 \mathrm{mg} / \mathrm{dl}$ y $11,2 \mathrm{mg} / \mathrm{dl}$ para el masculino (Fig. 1). La concentración de Lp (a) para los percentiles 50, 75 y 90 fue de 11,8, 28,8 y $48,5 \mathrm{mg} / \mathrm{dl}$ para el sexo femenino y $11,2,29,4$ y 51,1 $\mathrm{mg} / \mathrm{dl}$ para el sexo masculino.

Al distribuir el sexo masculino por grupos etarios con un rango de cinco años no se observó diferencia significativa en los niveles de Lp(a) entre cada grupo, sin embargo, en el sexo femenino se evidenció un nivel mayor de Lp (a) (aunque estadísticamente no significativo) en el grupo de 40-44 años (Fig. 1), con una mediana de $20,9 \mathrm{mg} / \mathrm{dl}$, lo que condujo a re-clasificar la población femenina en dos sub-grupos: mujeres menores 40 años $(n=93)$ y mujeres de 40 y más años $(n=219)$, que al ser comparados presentaron diferencias estadísticamente significativas en los niveles de Lp (a): mediana $13 \mathrm{mg} / \mathrm{dl} v s .16 \mathrm{mg} / \mathrm{dl}$ respectivamente; $\mathrm{p}<0,02$. Al analizar el subgrupo de mujeres de 40 años y más y dividirlo según el antecedente de recibir o no terapia hormonal de reemplazo $(\mathrm{TRH})$ se encontró que el grupo con TRH ( $\mathrm{n}=$ 91) presentó una concentración de Lp (a) significativamente menor (mediana: $8,5 \mathrm{mg} / \mathrm{dl}$ ) que aquellas que no recibían

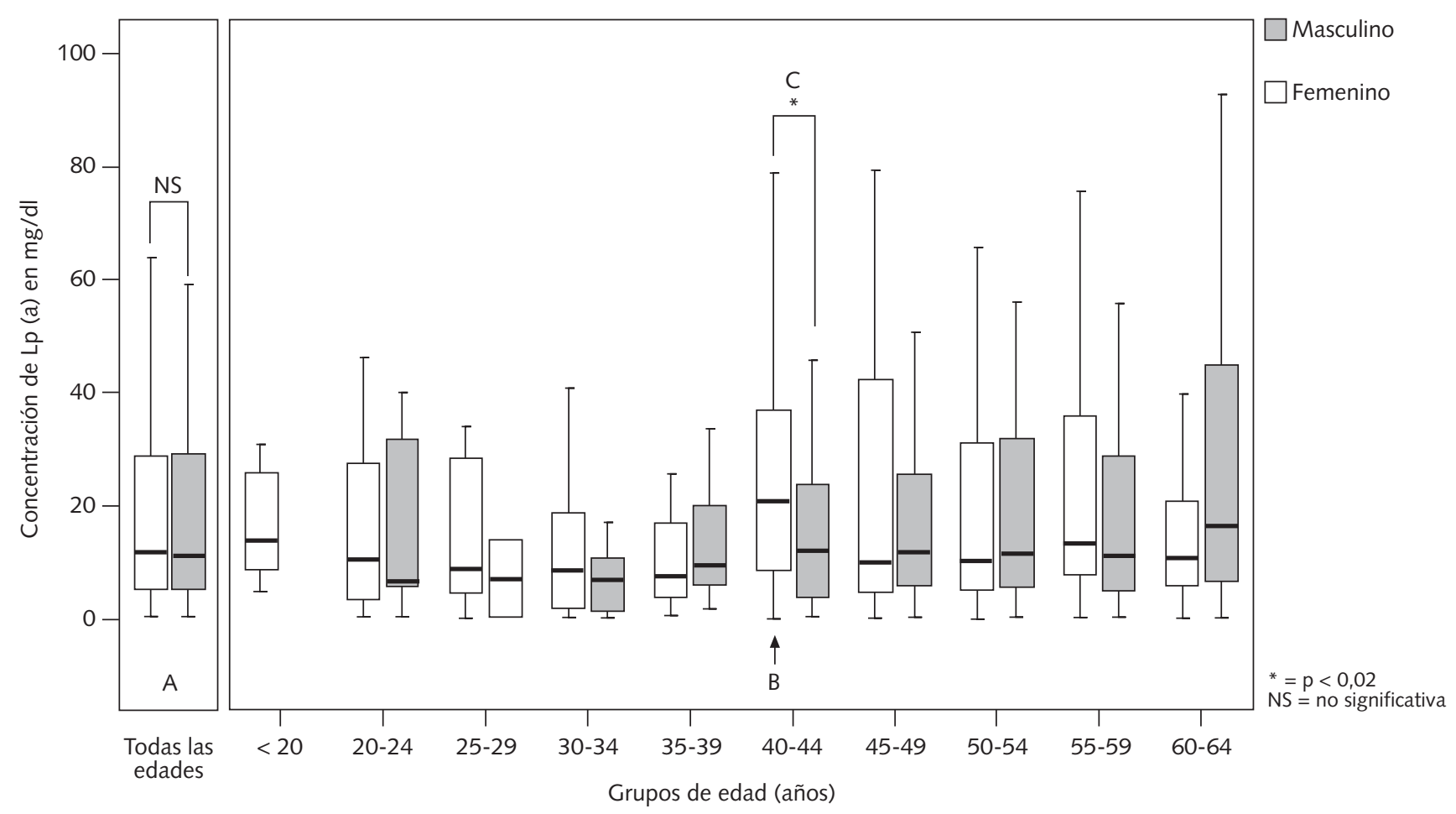

Fig. 1. Niveles de Lp (a) sérica según edad y sexo en individuos de la ciudad de Maracaibo. 


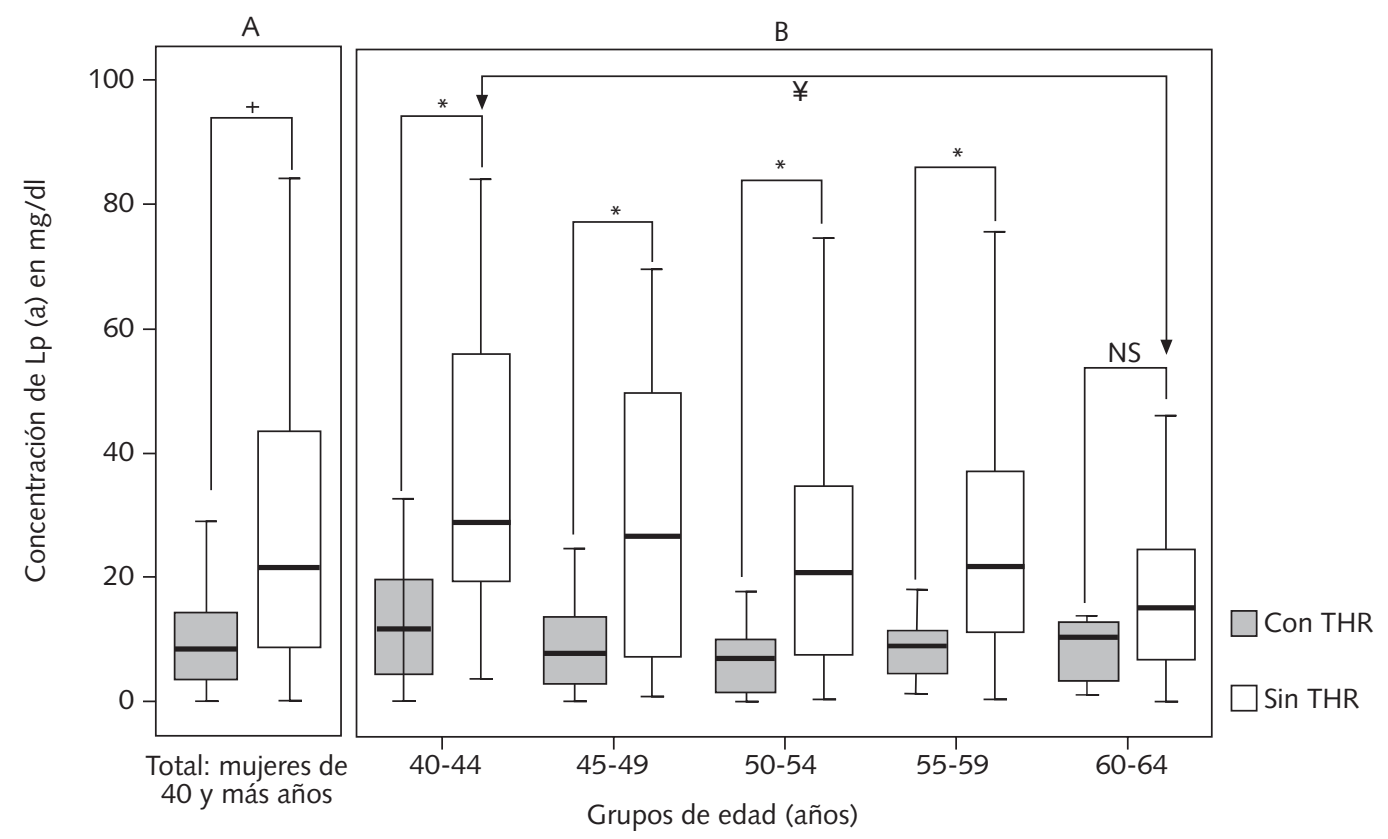

$+=p<0,01 ;{ }^{*}=p<0,01 ; ¥=p<0,03$ entre los grupos de 40-44 y 60-64 años sin TRH (previa transformación logarítmica, ANOVA y Tukey test post Hoc); NS = no significativa

Fig. 2. Comportamiento de la $L p($ a) en mujeres con y sin terapia hormonal de reemplazo.

TRH ( $\mathrm{n}=128$; mediana: $21,5 \mathrm{mg} / \mathrm{dl}$ ) p $<0,001$ (Fig. 2) (A). Este hallazgo llevó a la descomposición de estos dos grupos según la edad con un rango de 5 años, donde se observó que aquellas que recibían TRH siempre exhibieron una concentración de Lp (a) menor que aquellas sin TRH ( $p<0,01)$, salvo en el grupo de 60-64 años donde no hubo diferencia. Sin embargo, el grupo que no recibió TRH presentó una suave caída en los niveles de Lp (a) que se hace significativa al comparar los grupos etarios extremos, es decir, 40-44 años vs. 60-64 años (Fig. 2).

\section{DISCUSIÓN}

La asociación entre los niveles plasmáticos de Lp (a) y la enfermedad cardiovascular aterosclerótica ha sido documentada ampliamente en los últimos 20 años $(1,2)$. El papel fisiológico de la Lp (a) es aún desconocido, sin embargo, en la actualidad se conocen en profundidad varios de los procesos que relacionan esta lipoproteína con el fenómeno de aterotrombosis y que tienen relación con su semejanza a la LDL, la similitud entre la apoproteína (a) y el plasminógeno, la inducción de la proliferación de células musculares lisas y el estímulo de la síntesis del inhibidor tisular del plasminógeno tipo 1 (4-6).

Se admite que la concentración de Lp (a) es poco influenciada por el sexo, lo cual se corresponde con los resultados generales obtenidos en nuestro estudio ya que no se encontró diferencia significativa al comparar esta variable para todas las edades, evidenciándose la misma heterogeneidad (valores comprendidos entre 0,1-99 $\mathrm{mg} / \mathrm{dl}$ ) y una distribución no normal citada en otras investigaciones $(1,2)$. Sin embargo, en la distribución para los diferentes grupos etarios se evidenció un hallazgo importante: Los niveles de Lp (a) en el sexo femenino para el grupo de 40-44 años fueron superiores a su contraparte masculina (Fig. 1; p < 0,02), e incluso a los demás grupos de mujeres (Fig. 1, diferencia no significativa). Esto es consistente con un estudio realizado en individuos africanos de Nigeria en el que la población femenina entre los 45-54 años de edad presentó niveles significativamente mayores de Lp (a) que su contraparte masculina (7). De hecho, la posterior sub-división de las mujeres en menores de 40 años y mayores de 40 puso en evidencia la influencia de la perimenopausia, menopausia y la THR sobre el nivel de Lp (a), ya que las mujeres que usaban algún tipo de THR presentaban niveles inferiores de Lp (a) con respecto a las que no. En este sentido, la influencia de THR sobre esta lipoproteína se ha documentado extensamente (8). Por ejemplo, en un estudio reciente investigadores de la Universidad de Turquía determinaron el efecto de la administración de $17 \beta$-estradiol sobre los niveles de Lp (a) en 49 mujeres con menopausia quirúrgica, encontrando una significativa reducción de los niveles de esta lipoproteína especialmente en quienes mostraban niveles elevados antes del inicio del tratamiento (9). Lamentablemente, una limitación de nuestro estudio fue la imposibilidad de comparar las distintas opciones de THR debido a que la mayoría de las mujeres recibían solo estrógenos conjugados y una pequeña porción se distribuyó entre Tibolona o diferentes tipos de estrógenos más progestágenos diversos. 
La contribución más importante de esta investigación es que ha puesto en evidencia dos fenómenos que no habían sido reportados en la literatura. En primer lugar, la administración de TRH produce un efecto consistente sobre la concentración de Lp (a) para todas las edades estudiadas (por encima de los 40 años), manteniéndola dentro del límite normal sin diferencia con las mujeres en edad fértil. En segundo lugar, las mujeres que no recibían TRH presentaron niveles de Lp (a) evidentemente mayores al inicio de la perimenopausia/menopausia, es decir alrededor de los 40-44 años, pero que fueron disminuyendo de forma significativa hasta hacerse idénticos a los de las mujeres con TRH y de la misma edad alrededor de los 60 años. Esto sugiere que la deprivación estrogénica característica de la menopausia tiene un efecto transitorio sobre el nivel de la Lp (a) que tiende desaparecer alrededor de los 65 años. Es posible que la caída rápida de los estrógenos durante los primeros años de la menopausia se acompañe de cambios en el patrón de expresión de la apoproteína (a) y su ensamblaje hepático a la Apo-B100 así como a una disminución en la can-

\section{Bibliografía}

1. Berg K. Lp (a) lipoprotein: An overview. Chemist and Physics of lipids 1994; 67: 9-16.

2. Enríquez L, Matas P: Lipoproteína (a): Fisiología y Consideraciones Clínicas y Terapéuticas. Med Clin (Barc) 2001; 116: 746-9.

3. Sorell L, Rojas G, Rodríguez M. A Sandwich ELISA based on antiApo(a) and anti Apo-B monoclonal antibodies for lipoprotein(a) measurement. Clinical Chemical Acta 1995; 236: 59-70.

4. Graninger D, Kirschenlohr H. Proliferation of human smooth muscle cells promoted by Lp (a). Science 1993; 260: 1655-58.

5. Kojima S, Harpel P, Rifkin D. Lipoprotein(a) Inhibits the Generation of Transforming Growth Factor b: An Endogenus inhibitor of Smooth Muscle Cell Migration. Journal of Cell Biology 1991; 113: 1439-45

6. Hancock MA, Boffa MB, Marcovina SM, Nesheim ME, Koschinsky, ML. Inhibition of plasminogen activation by lipoprotein (a): Critical tidad de receptores hepáticos para la LDL (receptores para ApoB/E), produciendo un incremento agudo en la concentración de la Lp (a), que posteriormente es compensado bien sea por cambios atribuibles al proceso de envejecimiento (disminución de la síntesis y ensamblaje de las partículas lipoproteicas) o a cambios relativos en la relación estrógenos/andrógenos durante la menopausia tardía, ya que en estudios realizados en mujeres post-menopáusicas que recibieron esteroides anabolizantes se ha observado una disminución significativa de los niveles de Lp (a) (10). Se deberán realizar estudios prospectivos que permitan aclarar la influencia de los distintos agentes hormonales utilizados en la TRH, incluyendo a los moduladores de los receptores de estrógenos sobre los niveles de Lp (a) y su relación con el riesgo de padecer eventos cardiovasculares agudos. Igualmente, debido a la riqueza étnica de Venezuela, sugerimos su estudio en los diversos núcleos indígenas y Afro-Americanos de nuestra geografía con la finalidad de tener una mejor comprensión de su comportamiento epidemiológico. domains in apolipoprotein(a) and mechanism of inhibition on fibrin and degraded fibrin surfaces. J Biol Chem 2003; 278: 23260-69.

7. Evans R, Bunker K, Ukoli F, Küller L. Lipoprotein (a) distribution in a Nigeria Population. Ethn Healt 1997; 2: 47-58.

8. Jenner J, Ordovas J, Lamon S, Schaefer M, Wilson P, Castelli W, et al. Effects of age, sex, and menopausal status on plasma lipopreotein (a) levels. The Framingham offdpring Study. Circulation 1993; 87: 1135-41.

9. Kiran H; Kiran G; Ekerbicer HC, Kilinc M. Effects of intranasal estradiol treatment on serum lipoprotein (a) and lipids in hysterectomized women. Gynecol Obstet Invest 2004; 57: 191-5.

10. Soma M, Meschia M, Bruchi F, Morrisett J, Paoletti R, Fumagalli R, et al. Homonal agents used in lowering lipoprotein (a). Chemistry Physic Lipids 1994; 67: 345-50. 